[Agr. Biol. Chem., Vol. 34, No. 10, p. $1590 \sim 1592,1970$ ]

\title{
1solation and Identification of Indole Derivatives in Clubroots of Chinese Cabbage
}

Sir:

It has well been known that roots of Chinese cabbage, Brassica pekinensis Rupr. infected with Plasmodiophora brassicae Woronin enlarge to form "clubs." Up to now, however, the cause of this malformation has not been clarified. On the other hand, Katsura et al. ${ }^{1,21}$ paperchromatographically demonstrated the presence of two unidentified neutral compounds with auxin activity in clubroots of Brassicae rapa var. neosuguki Kitam. infected with the same kind of fungus. Similarly, Kavanagh et al. ${ }^{33}$ conducted a qualitative chromatographic analysis for indole auxins in Plasmodiophora brassicae-infected cabbage hypocotyls, resulting in the detection of indoleacetonitrile (I). Here we wish to report the isolation and identification of I, I-methoxyindoleacetonitrile (II), 4-methoxyindoleacetonitrile (III), methyl indoleacetate (IV) and indoleacetamide (V) from clubroots of Chinese cabbage. Among them, II and III are the first compounds whose presence has been confirmed in higher plants.

At first, acidic and neutral ethyl acetatesoluble fractions were preliminarily separated from healthy and diseased roots of Chinese cabbage and applied to preparative paper chromatography (isopropanol-ammonium hydroxide-water, $10: 1: 1$ ), followed by the

1) K. Katsura, H. Egawa, T. Toki and S. Ishii, Ann. Phytopath. Soc. Japan, 32, 123 (1966).

2) K. Katsura, H. Egawa, M. Masuko and A. Ueyama, Proc. Kansai Plant Protection Soc., No. 11, 23 (1969).

3) J. A. Kavanagh, M. N. Reddy and P. H. Williams, Phytopathol., 59, 1035 (1969).
Avena coleoptile straight growth test. Hardly any biological activity was observed in the histograms of the acidic fractions from healthy and diseased plants. On the other hand, distinctly different patterns were observed between the neutral fractions from the both origins. Remarkable biological activity was detected at $R f \quad 0.7 \sim 0.9$ in the histogram of the diseased-neutral fraction. Then, isolation of active compounds was attempted in the following way.

The neutral ethyl acetate-soluble fraction $(6 \mathrm{~g})$ obtained from clubroots $(30 \mathrm{~kg})$ harvested in 1969 in Ibaragi Prefecture was chromatographed on a silica gel column using hexaneethyl acetate. The eluate with hexane containing $10 \%$ ethyl acetate gave II, and that with $15 \%$ ethyl acetate afforded I, III and IV, which were subsequently separated from each other by preparative thin-layer chromatography using Silica Gel $\mathrm{GF}_{254}$, hexane-ethyl acetate $(4: 1)$ and benzene-isopropyl ether $(4: 1)$. The isolation procedure is shown in Fig. 1. Moreover, the neutral $n$-butanol-soluble fraction obtained after the separation of the abovementioned ethyl acetate-soluble fraction was chromatographed on a silica gel column. Elution with ethyl acetate gave V. Yields of I, II, III, IV and V were 20, 10, 5, I and $1 \mathrm{mg}$, respectively.

Mass spectrometry of I gave a parent peak at m/e 156. Coloration with the Ehrlich's reagent as well as the UV spectrum suggested the presence of indole nucleus. $\lambda_{\max }^{\mathrm{EtOH}} \mathrm{m} \mu$ $(\log \varepsilon): 218$ (4.43), 270 (3.93), 278 (3.92), 289 (3.66); IR $\nu_{\text {xaax }}^{\text {Nujol }} \mathrm{cm}^{-1}: 3480(\mathrm{NH}), 2240(\mathrm{CN})$; NMR (in $\left.\mathrm{CDCl}_{3}\right) \delta: 3.80\left(2 \mathrm{H}, \mathrm{s}, \mathrm{CH}_{2}\right)$. These 


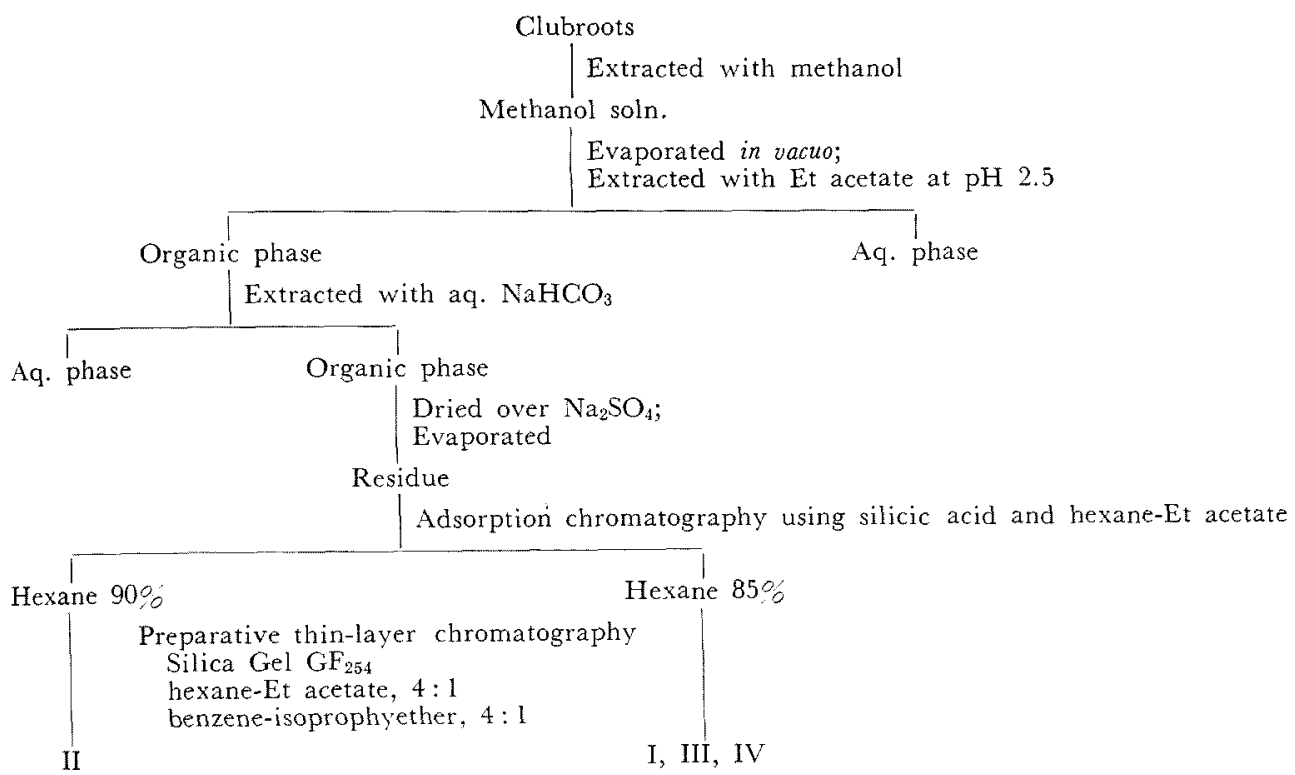

FIG. 1. Isolation Procedure of Indole Derivatives from Clubroots of Chinese Cabbage.

data are completely identical with those of the authentic sample of indoleacetonitrile.

The molecular weight of II was revealed to be 186 by mass spectrometry. The presence of indole nucleus in II was similarly indicated. $\lambda_{\max }^{\mathrm{E}+\mathrm{H}} \mathrm{m} \mu(\log \varepsilon): 218(4.42), 270(3.70), 286$ (3.66), $297(3.58) ; \nu_{\max }^{\text {Nujol }} \mathrm{cm}^{-1}: 2240(\mathrm{CN})$, no $\mathrm{NH}$; NMR (in $\mathrm{CDCl}_{3}$ ) $\delta: 3.78\left(2 \mathrm{H}, \mathrm{s}, \mathrm{CH}_{3}\right), 4.07$ $\left(3 \mathrm{H}, \mathrm{s}, \mathrm{OCH}_{3}\right) ; m / e 171\left(\mathrm{M}-\mathrm{CH}_{3}\right), 155(\mathrm{M}-$ $\left.\mathrm{OCH}_{3}\right)$. The methoxyl group can be attached to either 1- or 3-position in the indole nucleus. The chemical shifts of methoxyl and methylene $\left(-\mathrm{CH}_{2} \mathrm{CN}\right)$ are too low if they are presumed to be located together at $\mathrm{C}-3$ to form 3-cyanomethyl-3-methoxyindolenine. ${ }^{4,5}$ Thus the structure of II was established as 1-methoxyindoleacetonitrile.

III was shown to be an indole derivative with the molecular weight 186 based on the mass spectrometry, color reaction and UV spectrum.

4) L. A. Cohen, J. W. Daly, H. Kny and B. Witkop, J. Am. Chem. Soc., 82, 2184 (1960).

5) "NMR Spectra Catalog," ed. by Varian Associates, Spectrum No. 231 and 286 (1962). $\lambda_{\mathrm{met} x}^{\mathrm{EtOH}} \mathrm{m} \mu(\log s): 220(4.64), 265$ (3.86), 278 (3.73), 289 (3.71); $\nu_{\max }^{\mathrm{Nujol}} \mathrm{cm}^{-1}: 3480(\mathrm{NH}), 2240(\mathrm{CN})$; NMR (in $\mathrm{CDCl}_{3}$ ) $0: 3.95\left(3 \mathrm{H}, \mathrm{s}, \mathrm{OCH}_{3}\right), 4.05(2 \mathrm{H}$, $\left.\mathrm{s}, \mathrm{CH}_{2}\right) ; m / e 171,155$. By comparing the NMR spectrum of III with those of methyl chloroindoleacetates, ${ }^{6}$ each containing a chlorine atom at C-4, 5, 6 or 7, the methoxyl group in III was assigned to $\mathrm{C}-4$. Therefore, 4methoxyindoleacetonitrile ${ }^{7}$ was synthesized starting from 2-amino-6-nitrotoluene ${ }^{81}$ and shown to be completely identical with III on the basis of UV. IR, NMR and mass spectra.

Identification of IV as methyl indoleacetate was accomplished by comparison with the authentic specimen of the mass spectra as well as of the behaviors on thin-layer chromatography(hexane-ethyl acetate, $4: 1$ or ben-

6) We wish to express our heartiest thanks to Dr. S. Marumo of Nagoya University for providing the NMR data of these compounds.

7) T. R. Govindachari, P. M. Pillai, K. Nagarajan and N. Viswanathan, Tetrahedron, 21, 2957 (1965).

8) 4-Methoxyindoleacetonitrile was synthesized by Mr. M. Nagao of our laboratory to whom we are grateful. 
zene-isopropyl ether, $4: 1$ ).

$\mathrm{V}$ was proved to be identical with the authentic indoleacetamide based on mass spectrometry and thin-layer chromatography (dichloromethane-methanol, 10:1 or isopropyl ether-acetic acid, 9:1).

The biological activities of the new indole derivatives, II and III were assayed using the Avena coleoptile straight growth test. Contrary to the expectation, II showed slight activity and III was almost inactive. These indoleacetonitriles including I are supposed to be the metabolites of the diseased roots and not of the causal fungus, since glucobrassicin and neoglucobrassicin or 1-methoxyglucobras- sicin have been isolated by Gmelin et al, ${ }^{9,101}$ as normal constituents characteristic of the Brassica family.

Isolation of other growth substances in clubroots of Chinese cabbage is in progress.

Michio Nомото

Saburo TAMURA

Department of Agricultural Chemistry

The University of Tokyo, Bunkyo-ku, Tokyo

Received August 11, 1970

9) R. Gmelin, M. Saarivirta and A.I. Virtanen, Suomen Kemist., B 33, 172 (1960) [C. A., 55, 8384 (1961)].

10) R. Gmelin and A. I. Virtanen, ibid., B 35, 34 (1962) [C. A., 57, 16534 (1962)]. 